\title{
Photophysical Properties of Acene DCDHF Fluorophores: Long-Wavelength Single-Molecule Emitters Designed for Cellular Imaging
}

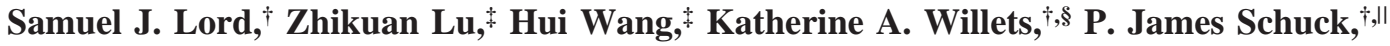 \\ Hsiao-lu D. Lee, ${ }^{\dagger}$ Stefanie Y. Nishimura, ${ }^{\dagger}$ Robert J. Twieg, ${ }^{*}$ and W. E. Moerner*, ${ }^{\dagger}$ \\ Department of Chemistry, Stanford University, Stanford, California 94305-5080, and \\ Department of Chemistry, Kent State University, Kent, Ohio 44240
}

Received: February 14, 2007; In Final Form: June 19, 2007

\begin{abstract}
We report the solvatochromic, viscosity-sensitive, and single-molecule photophysics of the fluorophores DCDHF-N-6 and DCDHF-A-6. These molecules are members of the dicyanomethylenedihydrofuran (DCDHF) class of single-molecule emitters that contain an amine electron donor and a DCDHF acceptor linked by a conjugated unit; DCDHF-N-6 and DCDHF-A-6 have naphthalene- and anthracene-conjugated linkers, respectively. These molecules maintain the beneficial photophysics of the phenylene-linked DCDHF (i.e., photostability, emission wavelength dependence on solvent polarity, and quantum yield sensitivity to solvent viscosity), yet offer absorption and emission at longer wavelengths that are more appropriate for cellular imaging. We demonstrate that these new fluorophores are less photolabile in an aqueous environment than several other commonly used dyes (rhodamine 6G, Texas Red, and fluorescein). Finally, we image single copies of the acene DCDHFs diffusing in the plasma membrane of living cells.
\end{abstract}

\section{Introduction}

Applying single-molecule techniques to biological problems is currently an active and growing field of research. ${ }^{1-3}$ Exploring biological activity within cells one molecule at a time makes it possible to detect hidden processes previously not seen or understood. It also becomes possible to reveal physiologically relevant heterogeneities obscured by the ensemble averaging of bulk measurements. ${ }^{4-8}$ However, these advantages can be realized only when using suitable fluorescent probes, and so there is a general effort to develop new and better emitters. ${ }^{9-13}$ For example, there is a need for small organic fluorophores that are brighter, more photostable, excitable in the green or red (in order to reduce cellular autofluorescence background signals), and ideally report a change in the environment with a corresponding detectible change in fluorescence. Here, we report the solvatochromic and photophysical properties of two red-emitting members of the dicyanomethylenedihydrofuran (DCDHF) class of single-molecule fluorophores currently being developed by our labs. (The nomenclature we use in this paper is "acceptorlinker-donor," where the acceptor is the DCDHF structure, the linker is a conjugated ring-either phenylene $\mathrm{P}$, naphthalene $\mathrm{N}$, or anthracene $\mathrm{A}$-and the donor is an amine with two alkyl chains with six carbons each. Note that this deviates slightly from previous nomenclature in which the phenyl linker was not specifically assigned, i.e., DCDHF-P-6 was formerly labeled "DCDHF-6.")

As reported previously, ${ }^{14-17}$ nonlinear optical chromophores that contain an amine donor and a DCDHF acceptor connected

* Author to whom correspondence should be addressed. E-mail: wmoerner@stanford.edu.

† Stanford University.

$\doteqdot$ Kent State University.

$\S$ Present address: Department of Chemistry and Biochemistry, The University of Texas, Austin, TX 78712.

"Present address: Molecular Foundry, Lawrence Berkeley National Laboratory, Berkeley, CA 94720. by a conjugated linker not only are robust single-molecule fluorophores but also exhibit sensitivity to solvent polarity and viscosity (local rigidity). The most extensively studied derivative from this class is DCDHF-P-6, whose donor and acceptor are conjugated by a 1,4-substituted phenyl ring (see Figure 1A): ${ }^{16}$ DCDHF-P-6 shows minimal fluorescence in solution-the result of an intramolecular twist on the excited-state manifold ${ }^{16,18,19}$ that introduces nonradiative relaxation pathways - but becomes very bright when constrained in rigid environments, when the nonradiative conformer is inhibited from forming. The primary benefit of this viscosity sensitivity is a fluorogenic label that brightens dramatically ("turns on") upon binding to cellular components, producing an enhanced signal relative to the background fluorescence from unbound fluorophores. DCDHFP-6, however, is not ideal as a cellular label because it requires illumination at short wavelengths that also produce high background signal from cell autofluorescence.

To refine our probes for cellular imaging, it is necessary to retain the beneficial properties of DCDHF-P-6 while pushing absorption to longer wavelengths (>500 nm), where endogenous molecules such as flavins that contribute to cellular autofluorescence do not absorb. ${ }^{20}$ The most straightforward way to redshift a dye is to lengthen its conjugation, so we have been developing several DCDHF derivatives with different conjugated linkers. For instance, one of the originally studied DCDHFs extended the conjugation using a styryl linker, ${ }^{15}$ which redshifted the absorption and emission but also lowered the fluorescence quantum yield $\Phi_{\mathrm{F}}$ in solution (presumably by increasing the number of accessible twisted states by adding additional isomerization pathways). An alternative modification that red-shifts the absorption without adding additional nonradiative pathways extends the conjugation using acenes, which are more rigid than styryl groups. ${ }^{21}$ Here, we report that the derivatives DCDHF-N-6, which contains a 2,6-naphthyl linker between the donor and acceptor, and DCDHF-A-6, with a 2,6anthryl linker (see Figure 1), not only maintain the solvato- 
A
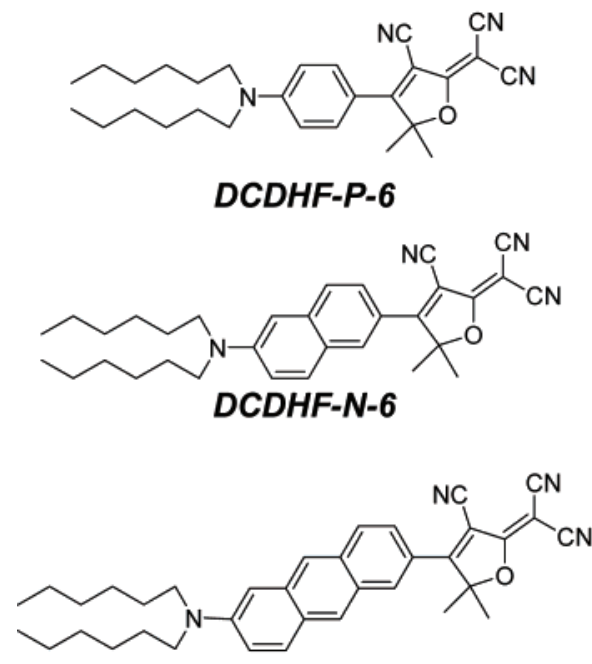

DCDHF-A-6
B

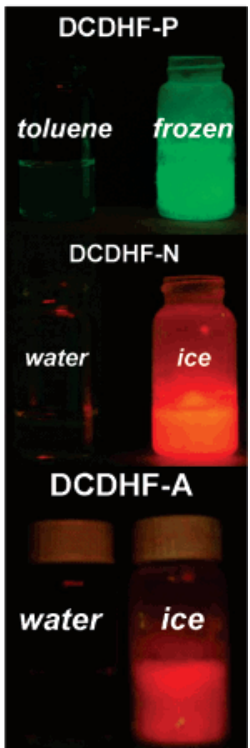

Figure 1. (A) Structures of DCDHF-P-6, DCDHF-N-6, and DCDHFA-6. (B) Equal concentrations of fluorophore in liquid (left) and frozen (right) solvent solutions illuminated by a handheld UV lamp $(365 \mathrm{~nm})$, with a $500 \mathrm{~nm}$ long-pass filter placed before the lens of a digital camera in order to remove scattered excitation light and record only the fluorescence. In the rigid environment of the frozen solvents, emission dramatically increases. This fluorescence jump upon increase in local rigidity is characteristic of the entire class of DCDHF fluorophores and occurs in a range of solvents.

chromism and viscosity sensitivity characteristic of their phenylcontaining sister DCDHF-P-6 but also can be excited at 514 and $532 \mathrm{~nm}$ or longer (where cellular autofluorescence background is drastically suppressed). ${ }^{20,22}$

In a previous publication, the naphthyl DCDHF was used as a membrane probe: single copies were studied as lipid analogs to probe diffusion in the plasma membrane of eukaryotic cells. ${ }^{22}$ Here, we report the fundamental photophysical properties and environmental dependence of two acene DCDHFs in controlled conditions (e.g., in solvents with a range of polarities and in polymer films) in order to better judge and understand the full utility and potential of these probes.

\section{Materials and Methods}

2.1. Experimental Methods. Bulk solution absorption and emission spectra were acquired on a Perkin-Elmer Lambda 19 UV-vis spectrometer and a SPEX Fluoromax-2 fluorimeter using a standard $1 \mathrm{~cm}$ path length quartz cuvette. Fluorescence quantum yields were referenced against standards with known quantum yields, corrected for differences in optical density and solvent refractive index. ${ }^{23}$ DCDHF-P-6 and DCDHF-N-6 in toluene were measured against rhodamine $6 \mathrm{G}$ in ethanol $\left(\Phi_{\mathrm{F}}\right.$ $=0.95)$; the quantum yield of DCDHF-A-6 in toluene was measured against Texas Red in ethanol $\left(\Phi_{\mathrm{F}}=0.93\right) .{ }^{24}$ The fluorophores in other solvents were measured against their own values in toluene. All quantitative measurements were done at low concentrations (absorbance values less than 0.2 ) to avoid any complications with dimer or aggregate formation, although evidence thereof was not encountered even at higher concentrations $\left(\sim 10^{-4} \mathrm{M}\right)$. Extinction coefficients were measured from dilutions of solutions with known concentrations.

For bulk spectra in polymer films, a small amount of highly concentrated dye solution was mixed into a $20 \%$ (by mass) solution of poly(methyl methacrylate) (PMMA, $T_{\mathrm{g}}=105^{\circ} \mathrm{C}$,
$\mathrm{MW}=75000 \mathrm{~g} / \mathrm{mol}$, atactic, polydispersity $\sim 2.8$, PolySciences Inc.) in toluene, then the solution was spin-cast onto a glass slide producing a film $20 \mu \mathrm{m}$ thick as measured by ellipsometry. For absorption spectra, the slide was placed orthogonal to the beam with an undoped film in the reference channel; for emission spectra, the slide was placed in an orientation so as to reject the scattered excitation light. Absorption and emission were measured at several locations throughout the film to compensate for macroscopic heterogeneity in film thickness and coverage, which otherwise would result in variations in absorbance and emission signals.

Fluorescence-lifetime experiments were performed using a home-built scanning-stage confocal microscope based upon a commercial inverted microscope (Nikon TE300). Samples were excited using $532 \mathrm{~nm}$ illumination from an optical parametric oscillator (Coherent Mira-OPO) pumped by a mode-locked Ti: sapphire laser (Coherent Mira 900) with a repetition rate of 75 $\mathrm{MHz}$ and pulses $120 \mathrm{fs}$ in duration; the Ti:sapphire was pumped by an Ar-ion laser (Coherent Innova 200). For two-photon fluorescence-lifetime measurements, $800 \mathrm{~nm}$ light was used directly from the mode-locked Ti:sapphire laser. Emitted photons passed through a $545 \mathrm{~nm}$ dichroic and two $545 \mathrm{~nm}$ long-pass filters and were detected with a single-photon avalanche photodiode (Micro Photon Devices PDM) equipped with a time-correlated single-photon counting data analysis board (PicoQuant TimeHarp200). The instrument response function (IRF) was recorded using scattered excitation light from a clean glass coverslip (the two long-pass filters were removed and OD filters were added to the beam path). The IRF was deconvolved from the data and then fit using a maximumlikelihood estimator.

Samples for quantitative single-molecule studies were prepared in 1\% (by mass) solutions of PMMA in distilled toluene doped with nanomolar fluorophore concentrations; these solutions were then spin-cast onto plasma-etched glass coverslips to produce films $30 \mathrm{~nm}$ thick as measured by ellipsometry. (The solvent distillation and plasma treatment of the substrates is done to remove spurious sources of fluorescence.) Samples were studied using a Nikon Diaphot 200 inverted microscope in an epifluorescence configuration ${ }^{25}$ using $532 \mathrm{~nm}$ light from a continuous wave doubled Nd:YAG laser (Spectra-Physics Millenia); the intensity at the sample was between 0.25 and $1.7 \mathrm{~kW} / \mathrm{cm}^{2}$. The emission was collected through a $100 \times, 1.4$ NA oil immersion objective, filtered using a $545 \mathrm{~nm}$ dichroic and $550 \mathrm{~nm}$ long-pass filter to remove scattered excitation light, and imaged onto a back-illuminated, frame-transfer Si CCD camera (Roper Scientific MicroMAX) with an integration time of $100 \mathrm{~ms}$.

Sequences of single-molecule images (i.e., movies) were used to extract the total number of detected photons before photobleaching, where all the photons (minus background) contributing to a single-molecule spot were spatially and temporally integrated. Even though the MicroMAX Si CCD does not have electron multiplication, the conversion gain (defined as the number of A-to-D converter counts per photoelectron) is 0.521 counts/electron for our camera; the number of detected photons is equal to ADC counts divided by conversion gain. It is also useful to calculate the number of emitted photons by correcting the measured value of detected photons using the collection efficiency of our setup ( $D=\eta_{\mathrm{Q}} F_{\text {coll }} F_{\text {opt }} F_{\text {filter }}$ ), which is the product of the camera quantum efficiency $\eta_{\mathrm{Q}}$, the angular collection factor $F_{\text {coll }}$ determined by the objective NA, the transmission factor through the objective and microscope optics $F_{\text {opt }}$, and the transmission factor through the various filters $F_{\text {filter }}$, 
SCHEME 1: Synthesis of DCDHF-A-6
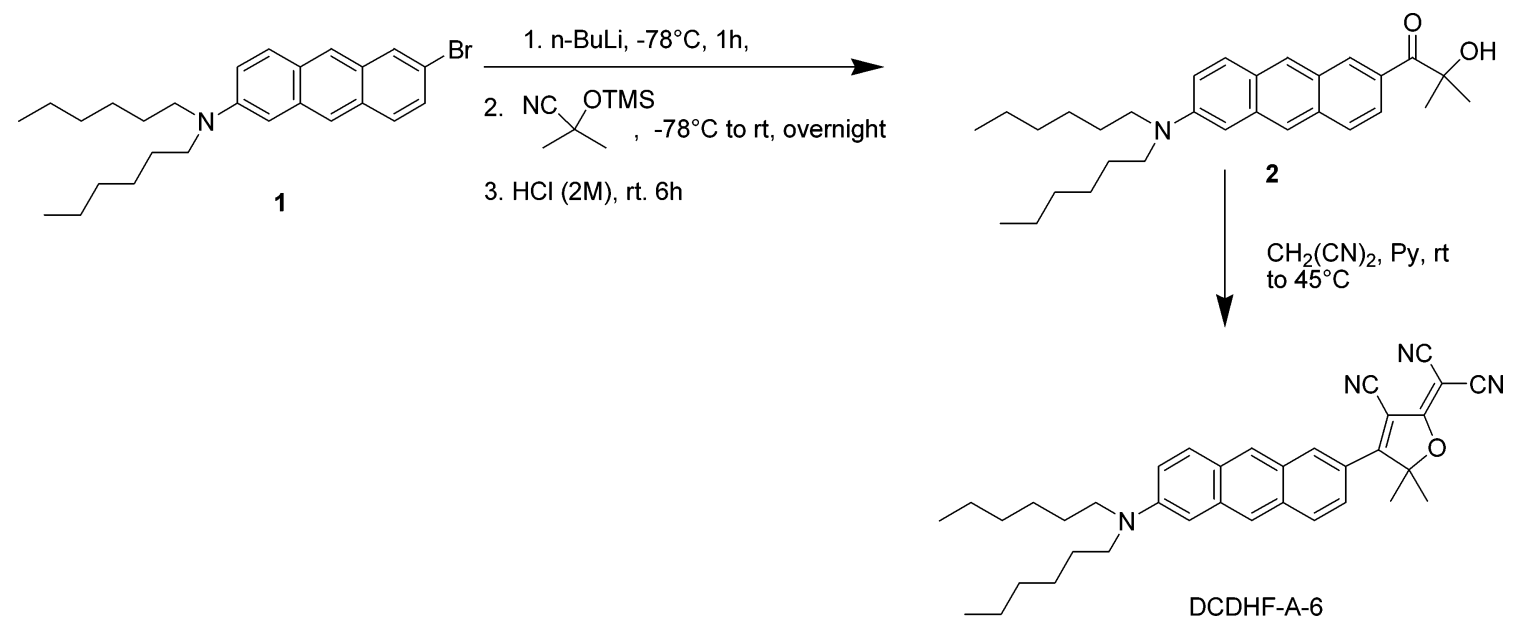

respectively. ${ }^{25}$ At the emission wavelengths, $\eta_{\mathrm{Q}}=75-80 \%$ for our camera, the maximum possible $F_{\text {coll }}$ for our setup is $38 \%$ for a single dipole emitter aligned horizontally; ${ }^{26}$ we measured $F_{\text {opt }}$ for our setup to be $50 \%$, and we measured $F_{\text {filter }}$ to be $60-$ $70 \%$ for the filter set we used. This yields $D_{\text {DCDHF-N-6 }}=9.1 \%$ and $D_{\text {DCDHF-A-6 }}=9.9 \%$.

Samples for aqueous bulk photostability measurements and single-molecule images were prepared using 5\% (by mass) gelatin (type A, Bloom 200, MP Biomedicals) in purified water. The gelatin solution was liquefied at $37{ }^{\circ} \mathrm{C}$. A small volume $(<0.5 \mu \mathrm{L})$ of dye stock solution in dimethyl sulfoxide was mixed with $10 \mu \mathrm{L}$ of gelatin, sandwiched between two glass coverslips, and allowed to gel at room temperature. Samples were imaged using the microscope and CCD camera setup described above. Fluorescein (unmodified parent version) was measured using a $488 \mathrm{~nm}$ laser (Novalux Protera 488-20), DCDHF-N-6 and Texas Red were imaged using $532 \mathrm{~nm}$, and DCDHF-A-6 was imaged using a $594 \mathrm{~nm}$ HeNe laser.

Movies of DCDHFs in the plasma membrane were recorded using a back-illuminated, frame-transfer, electron-multiplication Si EMCCD camera (Andor iXon or Roper Cascade). Samples were made by incorporating the probe into Chinese hamster ovary (CHO) cells as follows. A fluorophore film was made by drying $5 \mu \mathrm{L}$ of an $80 \mu \mathrm{M}$ solution of DCDHF-A-6 in chloroform onto a glass coverslip. This film was reconstituted with $50 \mu \mathrm{L}$ of ethanol; $4 \mu \mathrm{L}$ of this ethanol solution was added to $400 \mu \mathrm{L}$ of RPMI 1640 cell medium without phenol red (Gibco). The cells were incubated with $440 \mu \mathrm{L}$ of $\sim 80 \mathrm{nM}$ final dye/RPMI solution for $20 \mathrm{~min}$ at $37^{\circ} \mathrm{C}$. The solution was then aspirated and the cells were gently washed one time with RPMI 1640 medium which was then replaced by the $\mathrm{CHO}$ cell imaging buffer. (For further experimental details, components of the CHO imaging buffer, and imaging conditions for DCDHF-N6, see ref 22.)

2.2. Synthesis. Samples of DCDHF-P-6 (2-dicyanomethylene-3-cyano-5,5-dimethyl-4-(4'-dihexylaminophenyl)-2,5-dihydrofuran) and DCDHF-N-6 (2-dicyanomethylene-3-cyano-5,5dimethyl-4-[6-(dihexylamino)naphthalene-2-yl]-2,5dihydrofuran) were synthesized as reported elsewhere. ${ }^{22,27,28}$ The synthesis of DCDHF-A-6 (2-dicyanomethylen-3-cyano-5,5dimethyl-4-(6-dihexylaminoanthracen-2-yl)-2,5-dihydrofuran) has not been previously described and is summarized in Scheme 1 . The critical precursor 2-bromo-6-dihexylaminoanthracene $\mathbf{1}^{21}$ was lithiated with $n$-BuLi at $-78^{\circ} \mathrm{C}$ over $1 \mathrm{~h}$ and trapped with TMS-protected acetone cyanohydrin. The resulting $\alpha$-siloxyimine intermediate was hydrolyzed with $2 \mathrm{M}$ aqueous hydro- chloric acid at room temperature to afford the crude $\alpha$-ketol $\mathbf{2}$, which was used directly in the DCDHF ring preparation. By this route DCDHF-A-6 was prepared in overall $37 \%$ yield. ${ }^{29}$

A measured amount of 2-bromo-6-dihexylaminoanthracene $(0.42 \mathrm{~g}, 0.95 \mathrm{mmol})$ was added with $15 \mathrm{~mL}$ of anhydrous THF to a $100 \mathrm{~mL}$ round-bottom flask. The mixture was cooled to $-78{ }^{\circ} \mathrm{C}$, and $n$-BuLi in hexane $(2.5 \mathrm{M}, 0.46 \mathrm{~mL}, 1.15 \mathrm{mmol})$ was added with stirring over $10 \mathrm{~min}$. The resulting mixture was stirred for an additional $1 \mathrm{~h}$ at $-78^{\circ} \mathrm{C}$, and then 2-methyl-2trimethylsilyloxypropionitrile ${ }^{30}(0.80 \mathrm{~g}, 5.0 \mathrm{mmol})$ was added to the resulting mixture via syringe over $5 \mathrm{~min}$. After completion of addition, the temperature was allowed to increase gradually to room temperature and the reaction mixture was stirred overnight at room temperature. The reaction was quenched with $10 \mathrm{~mL}$ of $2 \mathrm{M}$ hydrochloric acid for $6 \mathrm{~h}$ and extracted with diethyl ether. The organic layer was washed with water and brine, and dried with anhydrous $\mathrm{MgSO}_{4}$. Solvent was removed by rotary evaporation giving the crude product, which was mixed with malononitrile $(2.0 \mathrm{~g}, 30 \mathrm{mmol})$, pyridine, $15 \mathrm{~mL}$, and 5 drops of acetic acid. This mixture was stirred for a day at room temperature, an additional day at $45^{\circ} \mathrm{C}$, and then poured into cold 5\% hydrochloric acid and extracted with dichloromethane. The organic solution was washed with water and brine and dried with anhydrous $\mathrm{MgSO}_{4}$. Solvent was removed by rotary evaporation, and the residue was purified by silica gel flash chromatography with hexane and hexane/ethyl acetate mixture (up to $5: 1$ ) as eluents to give $200 \mathrm{mg}$ of green solid (37\% yield in two steps). ${ }^{1} \mathrm{HNMR}\left(400 \mathrm{MHz}, \mathrm{CDCl}_{3}\right) \delta 8.60$ $(\mathrm{d}, J=2.0 \mathrm{~Hz}, 1 \mathrm{H}), 8.38(\mathrm{~s}, 1 \mathrm{H}), 8.06(\mathrm{~s}, 1 \mathrm{H}), 7.93(\mathrm{dd}, J=$ 9.3, 3.2 Hz, 2H), 7.81 (dd, $J=9.1,2.0 \mathrm{~Hz}, 1 \mathrm{H}), 7.27$ (dd, $J=$ 9.5, $2.4 \mathrm{~Hz}, 1 \mathrm{H}), 6.88(\mathrm{~d}, J=2.4 \mathrm{~Hz}), 3.48(\mathrm{t}, J=7.8 \mathrm{~Hz}$, $4 \mathrm{H}), 1.96(\mathrm{~s}, 6 \mathrm{H}), 1.77-1.67(\mathrm{~m}, 4 \mathrm{H}), 1.47-1.34(\mathrm{~m}, 12 \mathrm{H})$, $0.95(\mathrm{t}, J=7.0 \mathrm{~Hz}, 6 \mathrm{H}) .{ }^{13} \mathrm{C}$ NMR $\left(100 \mathrm{~Hz}, \mathrm{CDCl}_{3}\right) \delta 176.1$, $175.6,147.7,137.4,134.2,132.9,130.4$ (2C), 129.0, 127.2, 127.0, 122.0, 121.8, 121.0, 118.5, 112.1, 111.9, 111.1, 102.1, $98.5,97.5,57.0,51.3,31.7,27.5,27.3,26.8,22.7,14.1 . \mathrm{MS}$ (APCI) $\mathrm{m} / \mathrm{z}$ 545.0. UV-vis $\left(\mathrm{CH}_{2} \mathrm{Cl}_{2}\right) \lambda_{1}=296 \mathrm{~nm}, \epsilon_{1}=4.25$ $\times 10^{4} \mathrm{~L} \mathrm{~cm}^{-1} \mathrm{~mol}^{-1}, \lambda_{2}=378 \mathrm{~nm}, \epsilon_{2}=2.51 \times 10^{4} \mathrm{~L} \mathrm{~cm}^{-1}$ $\mathrm{mol}^{-1}, \lambda_{3}=397 \mathrm{~nm}, \epsilon_{3}=2.47 \times 10^{4} \mathrm{~L} \mathrm{~cm}^{-1} \mathrm{~mol}^{-1}, \lambda_{4}=619$ $\mathrm{nm}, \epsilon_{4}=3.38 \times 10^{4} \mathrm{~L} \mathrm{~cm}^{-1} \mathrm{~mol}^{-1}$. Anal. Calcd for $\mathrm{C}_{36} \mathrm{H}_{40} \mathrm{~N}_{4} \mathrm{O}$ : C, 79.38; H, 7.40; N, 10.29. Found: C, 79.00; H, $7.57 ; \mathrm{N}, 10.35$.

\section{Results and Discussion}

3.1. Bulk Photophysical Properties. As mentioned above, a key goal of this work is to identify derivatives in the DCDHF 


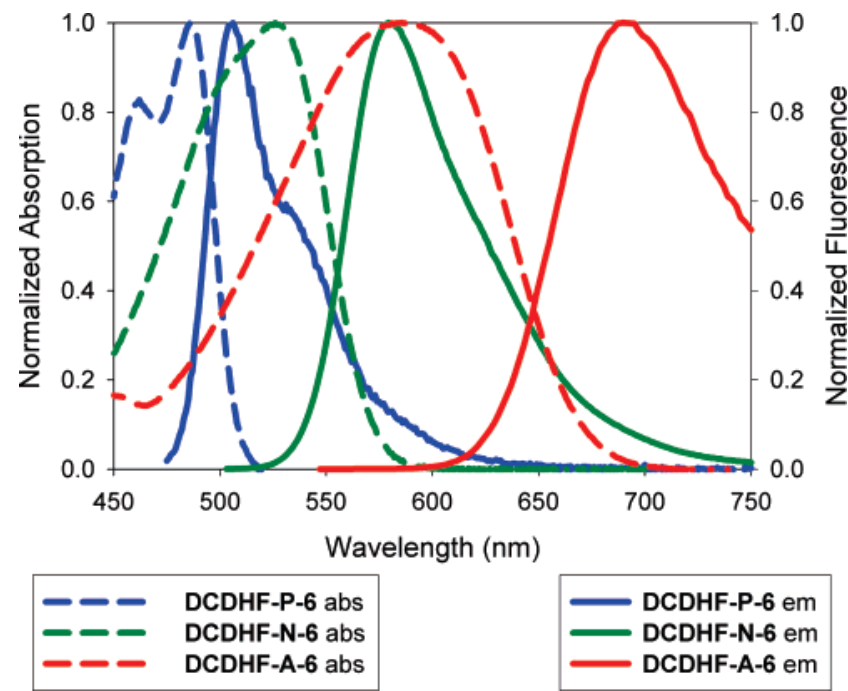

Figure 2. Normalized absorption and fluorescence emission spectra of DCDHF-P-6, DCDHF-N-6, and DCDHF-A-6 in toluene. Absorption for DCDHF-N-6 is at long enough wavelengths for excitation at 514 or $532 \mathrm{~nm}$, thus avoiding much of the cellular autofluorescence; DCDHF-A-6 can be excited at 532 or $594 \mathrm{~nm}$. Note also the enhanced Stokes shift of DCDHF-A-6 (104 nm) and DCDHF-N-6 (53 nm) over that of DCDHF-P-6 (21 nm).

class with long-wavelength absorption and emission where cellular autofluorescence is diminished. In most cases, pumping with wavelengths of $488 \mathrm{~nm}$ or shorter produces strong emission from essential cellular components such as flavins. ${ }^{20}$ As can be seen in Figure 2 and Table 1, in contrast to DCDHF-P-6, the new acene DCDHFs can be efficiently pumped using green or yellow light (e.g., $532 \mathrm{~nm}$ ) and exhibit large Stokes shifts, further enabling filtering to reject pumping light. Moreover, a full study of DCDHF-N-6 and DCDHF-A-6 solvent photophysics reveals that the reporter functions discovered in DCDHF-P-6 apply to the red-shifted dyes. Below we discuss the properties of these fluorophores that are beneficial for cellular imaging (including turn-on fluorescence upon rigidization, strong polarity sensitivity of emission wavelength and fluorescence lifetime, and bright emission), which make the red DCDHFs useful for various imaging modalities.

As is typical of many other DCDHF derivatives we have explored, the acene DCDHFs exhibit sensitivity to solvent viscosity: the fluorescence quantum yield increases with increasing local rigidity. For example, compare the quantum yields in solution and in polymer listed in Table 1 and note the increased brightness upon freezing solutions seen in Figure 1B. The previously proposed ${ }^{16}$ mechanism for this viscosity dependence involves a twist of the dicyanomethylene double bond, which introduces a nonradiative relaxation pathway via a twisted intramolecular charge-transfer (TICT) ${ }^{18}$ state. In solvents with low viscosity, the excited-state manifold is able to relax via bond twists to the TICT state, an increased electronic charge separation that is stabilized especially well by polar solvents (hence the lower quantum yield in polar solvents); in rigid environments, this twisting is kinetically less favorable, thus reducing the nonradiative contribution to the relaxation pathways and increasing the fluorescence quantum yield. This hypothesis was developed by performing Hartree-Fock and semiempirical quantum-chemistry calculations on the phenyl-conjugated DCDHF-P-6 derivative. ${ }^{16}$ Repeating these calculations on DCDHF-N-6 showed no significant departure from previous results (data not shown). While these calculations suggest that the sensitivity to local environment originates from the same intramolecular twist, they do not provide any insight into the enhanced quantum yield of the acene dyes over DCDHF-P-6; however, a mechanism could be revealed by a full photophysical study of the relaxation pathways, including a more extensive computational study.

Viscosity sensitivity for DCDHF-N-6 is quantified experimentally in the data in Table 2 and Figure 3A, which plots the logarithm of quantum yield versus the logarithm of solvent viscosity. Just as was previously seen with DCDHF-P-6, ${ }^{16}$ the alcohol solvents show a definite correlation between DCDHFN-6 emission probability and solvent viscosity, whereas other solvents do not exhibit a single trend. (As a side comment, we note that the high quantum yield of DCDHF-N-6 in toluene and benzene as compared to cyclohexane in Table 2 is observed to some degree in all DCDHF derivatives studied and is likely due to some specific solvent interaction-possibly $\pi-\pi$ stacking between the solvent and the fluorophore-that restricts intramolecular rotation pathways.)

The small viscosity range in Figure 3A covers only one decade, so it does not represent the large increase in quantum yield that occurs in more rigid environments: the plot is only a small portion of the dynamic range. Figure 1B better illustrates the dramatic jump in brightness as a solution of DCDHFs in liquid solvent is frozen to form a rigid environment. To partially

TABLE 1: Spectral Parameters of DCDHF-P-6, DCDHF-N-6, and DCDHF-A-6 in a Representative Range of Liquid Solvents ${ }^{a}$

\begin{tabular}{|c|c|c|c|c|c|c|}
\hline & solvent & $\Phi_{\mathrm{F}}$ & $\begin{array}{c}\epsilon_{\max } \\
\left(\mathrm{M}^{-1} \mathrm{~cm}^{-1}\right)\end{array}$ & $\begin{array}{c}\lambda_{\mathrm{abs}}^{\max } \\
(\mathrm{nm})\end{array}$ & $\begin{array}{c}\lambda_{\mathrm{em}}^{\max } \\
(\mathrm{nm})\end{array}$ & $\begin{array}{c}\mathrm{SM} \\
N_{\text {tot,emitted }}\end{array}$ \\
\hline DCDHF-P-6 & $\begin{array}{l}\text { PMMA } \\
\text { toluene } \\
\text { acetone } \\
\text { ethanol } \\
\text { water }\end{array}$ & $\begin{array}{l}0.92 \\
0.044 \\
0.0021 \\
0.0066 \\
0.002\end{array}$ & 71000 & $\begin{array}{l}486 \\
494 \\
469 \\
470\end{array}$ & $\begin{array}{l}507 \\
531 \\
528 \\
546\end{array}$ & $2.4 \times 10^{6}$ \\
\hline DCDHF-N-6 & $\begin{array}{l}\text { PMMA } \\
\text { toluene } \\
\text { acetone } \\
\text { ethanol } \\
\text { water }\end{array}$ & $\begin{array}{l}0.98 \\
0.85 \\
0.015 \\
0.017 \\
0.003\end{array}$ & $\begin{array}{l}47000 \\
36000 \\
38000\end{array}$ & $\begin{array}{r}534 \\
526 \\
533 \\
543 \\
\sim 550\end{array}$ & $\begin{array}{l}609 \\
579 \\
660 \\
657 \\
675\end{array}$ & $1.4 \times 10^{6}$ \\
\hline DCDHF-A-6 & $\begin{array}{l}\text { PMMA } \\
\text { toluene } \\
\text { acetone } \\
\text { ethanol } \\
\text { water }\end{array}$ & $\begin{aligned} & 0.71 \\
& 0.54 \\
& 0.023 \\
& 0.013 \\
&<0.001\end{aligned}$ & $\begin{array}{l}35000 \\
29000 \\
29000\end{array}$ & $\begin{array}{r}594 \\
585 \\
588 \\
602 \\
\sim 600\end{array}$ & $\begin{array}{r}686 \\
689 \\
826 \\
826 \\
>830\end{array}$ & $2.2 \times 10^{6}$ \\
\hline
\end{tabular}

\footnotetext{
${ }^{a} \Phi_{\mathrm{F}}$ is the fluorescence quantum yield, $\epsilon_{\max }$ is the maximum molar extinction coefficient, $\lambda_{\mathrm{abs}}{ }^{\text {max }}$ and $\lambda_{\mathrm{em}}{ }^{\text {max }}$ are the wavelengths of maximum absorption and emission, and SM $N_{\text {tot,emitted }}$ is the average total number of photons emitted per molecule before photobleaching measured moleculeby-molecule.
} 
TABLE 2: Photophysical and Solvatochromic Properties of DCDHF-N-6

\begin{tabular}{llcccccl}
\hline \multicolumn{1}{c}{ solvent } & $\Phi_{\mathrm{F}}$ & $\begin{array}{c}\lambda_{\text {abs }} \max \\
(\mathrm{nm})\end{array}$ & $\begin{array}{c}\lambda_{\mathrm{em}} \max \\
(\mathrm{nm})\end{array}$ & $n$ & $\epsilon_{\mathrm{r}}$ & $\Delta f$ & \multicolumn{1}{c}{$\begin{array}{c}\eta \\
\mathrm{cP})\end{array}$} \\
\hline water & 0.003 & 550 & 675 & 1.3330 & 78.5 & 0.3193 & 1.01 \\
acetonitrile & 0.014 & 543 & 664 & 1.3442 & 37.5 & 0.3053 & 0.345 \\
acetone & 0.015 & 533 & 660 & 1.3588 & 20.70 & 0.2843 & 0.316 \\
DMSO & 0.015 & 549 & 675 & 1.4770 & 47.2 & 0.2640 & 1.98 \\
THF & 0.18 & 529 & 636 & 1.4050 & 7.52 & 0.2096 & 0.55 \\
ethyl acetate & 0.21 & 523 & 623 & 1.3723 & 6.02 & 0.1997 & 0.455 \\
ethyl ether & 0.82 & 518 & 593 & 1.3526 & 4.335 & 0.1668 & 0.2332 \\
chloroform & 0.80 & 554 & 613 & 1.4459 & 4.806 & 0.1482 & 0.58 \\
isopropyl ether & 0.88 & 523 & 583 & 1.3673 & 3.88 & 0.1453 & 0.273 \\
dipropylamine & 0.10 & 528 & 591 & 1.4050 & 2.9 & 0.0826 & 0.53 \\
triethylamine & 0.021 & 516 & 569 & 1.4010 & 2.42 & 0.0477 & 0.363 \\
toluene & 0.85 & 526 & 579 & 1.4961 & 2.379 & 0.0134 & 0.59 \\
benzene & 0.88 & 525 & 581 & 1.5011 & 2.284 & 0.0030 & 0.652 \\
$n$-heptane & 0.31 & 514 & 537 & 1.3878 & 1.9 & -0.0034 & 0.55 \\
cyclohexane & 0.22 & 517 & 541 & 1.4266 & 2.023 & -0.0014 & 0.898 \\
& & & Alcohols & & & \\
methanol & 0.013 & 540 & 660 & 1.3288 & 32.63 & 0.3084 & 0.597 \\
ethanol & 0.017 & 543 & 657 & 1.3611 & 24.30 & 0.2886 & 1.2 \\
2-propanol & 0.048 & 543 & 651 & 1.3776 & 18.3 & 0.2729 & 2.4 \\
1-butanol & 0.068 & 546 & 649 & 1.3993 & 17.1 & 0.2625 & 2.948 \\
benzyl alcohol & 0.076 & 560 & 654 & 1.5396 & 13.1 & 0.2061 & 5.8
\end{tabular}

${ }^{a}$ Left columns, measured properties of fluorophore: fluorescence quantum yield $\left(\Phi_{\mathrm{F}}\right)$ and absorption and emission wavelengths. Right columns, solvent properties reported in refs 31 and 32: relative dielectric constant $\left(\epsilon_{\mathrm{r}}\right)$, index of refraction $(n)$, orientation polarizability $(\Delta f)$, and viscosity $(\eta)$. The excitation wavelength was $488 \mathrm{~nm}$. The $\Phi_{\mathrm{F}}$ of DCDHF-N-6 in toluene was referenced against rhodamine 6G in ethanol (0.95); all other solvents were measured against DCDHFN-6 in toluene. The rows are ordered by decreasing $\Delta f$.

quantify the full increase of fluorescence, we measured the quantum yield of DCDHF-N-6 in a PMMA polymer film to be nearly unity, while that in ethanol is only $1.7 \%$ (see Table 1). A similar increase in the fluorescence is demonstrated for DCDHF-A-6 in Table 1. We attribute this jump in brightness to the drastic change in viscosity, given that PMMA is still quite polar, approximately equivalent to dimethyl sulfoxide.

In solution, the acene fluorophores exhibit strong solvatochromism in addition to the viscosity sensitivity discussed above. Tables 2 and 3 list absorption and emission maxima, fluorescence quantum yield, and molar extinction coefficient $\left(\epsilon_{\max }\right)$ for DCDHF-N-6 and DCDHF-A-6 measured in various solvents. To explore the dependence of the Stokes shift on the solvent polarity, we use the Lippert formalism for general solvent polarity effects. ${ }^{23}$ Recalling that DCDHFs have an asymmetric donor-acceptor motif, it is reasonable to expect changes in the molecular dipole moment upon excitation: the charge-transfer state can be stabilized by solvent dipoles rearranging around the excited fluorophore; thus, the extent of stabilization depends on the polarity of the solvent. In the Lippert approximation the orientation polarizability $\Delta f$ is used as a parameter to represent the degree of molecular rearrangement around a dipole in a continuous medium leading to the observed Stokes shift:

$$
\begin{gathered}
\bar{v}_{\mathrm{A}}-\bar{v}_{\mathrm{F}}=\frac{2}{h c} \Delta f \frac{\left(\mu_{\mathrm{E}}-\mu_{\mathrm{G}}\right)^{2}}{a^{3}}+\text { constant } \\
\text { where } \quad \Delta f=\frac{\epsilon_{\mathrm{r}}-1}{2 \epsilon_{\mathrm{r}}+1}-\frac{n^{2}-1}{2 n^{2}+1}
\end{gathered}
$$

and $h$ is Planck's constant, $c$ is the speed of light in a vacuum, $a$ is the Onsager cavity radius (assumed to be $\sim 5 \AA$ ), and $\mu_{\mathrm{E}}$ and $\mu_{\mathrm{G}}$ are the excited- and ground-state dipole moments,
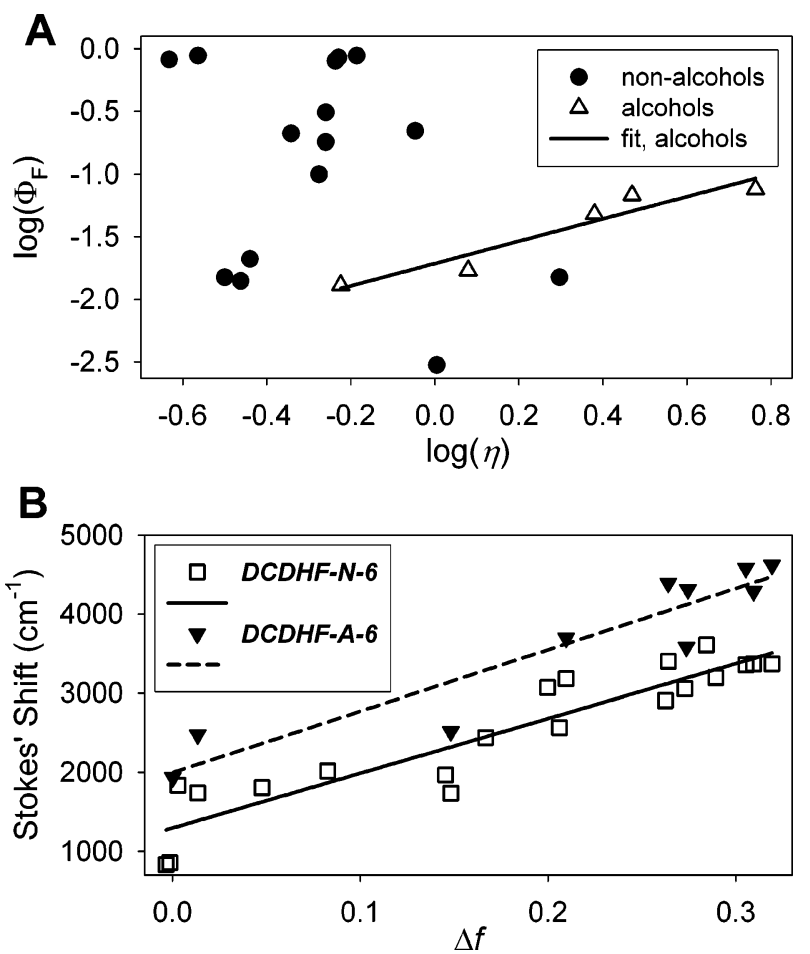

Figure 3. Environment-sensitivity spectroscopic data from Tables 2 and 3. (A) Log of fluorescence quantum yield as a function of the log of viscosity (relative to water $\eta=1.01 \mathrm{cP}$ ) for DCDHF-N-6. The data for the fluorophore in alcohols are fit by a line with slope of $0.89\left(R^{2}\right.$ $=0.91)$. The fluorophore does not exhibit this obvious trend in other solvents. (B) Lippert plot of emission Stokes shift vs the polarity parameter $\Delta f$ (see text for definition) for DCDHF-N-6 in all solvents in Table 2 and DCDHF-A-6 for a range of solvents. The data for DCDHF-N-6 are fit to a line with slope of $6921 \mathrm{~cm}^{-1}\left(R^{2}=0.86\right)$; the fit for the DCDHF-A-6 data has a slope of $7757 \mathrm{~cm}^{-1}\left(R^{2}=0.88\right)$.

TABLE 3: Solvatochromatic Properties of DCDHF-A-6 ${ }^{a}$

\begin{tabular}{lccccc}
\hline \multicolumn{1}{c}{ solvent } & $\begin{array}{c}\lambda_{\mathrm{abs}} \max \\
(\mathrm{nm})\end{array}$ & $\begin{array}{c}\lambda_{\mathrm{em}}{ }^{\max } \\
(\mathrm{nm})\end{array}$ & $n$ & $\epsilon_{\mathrm{r}}$ & $\Delta f$ \\
\hline water & 600 & 830 & 1.3330 & 78.5 & 0.3193 \\
acetonitrile & 586 & 801 & 1.3442 & 37.5 & 0.3053 \\
DMF & 597 & 804 & 1.4303 & 36.7 & 0.2745 \\
DMSO & 598 & 811 & 1.4770 & 47.2 & 0.2640 \\
THF & 586 & 748 & 1.4050 & 7.52 & 0.2096 \\
chloroform & 626 & 743 & 1.4459 & 4.806 & 0.1482 \\
toluene & 582 & 680 & 1.4961 & 2.379 & 0.0134 \\
hexane & 552 & 618 & 1.3751 & 1.89 & 0.0001 \\
& \multicolumn{5}{c}{ Alcohols } \\
methanol & 594 & 797 & 1.3288 & 32.63 & 0.3084 \\
1-propanol & 606 & 774 & 1.4101 & 13.9 & 0.2493
\end{tabular}

${ }^{a}$ Left columns, measured absorption and emission wavelengths. Right columns, solvent properties reported in refs 31 and 32: relative dielectric constant $\left(\epsilon_{\mathrm{r}}\right)$, index of refraction $(n)$, and orientation polarizability $(\Delta f)$. The rows are ordered by decreasing $\Delta f$.

respectively. The Lippert plots in Figure 3B show the Stokes shift versus $\Delta f$, and from the slopes we calculate the change in the dipole moment upon excitation to be $\left|\mu_{\mathrm{E}}-\mu_{\mathrm{G}}\right|=9.4 \mathrm{D}$ for DCDHF-N-6 and 9.7 D for DCDHF-A-6. These values are significantly greater than the value for DCDHF-P-6 (4.4 D); ${ }^{16}$ in other words, the acene derivatives exhibit stronger sensitivity to solvent polarity than their phenyl sister. Moreover, because DCDHF-N-6 and DCDHF-A-6 exhibit red-shifts in emission of over $100 \mathrm{~nm}$ going from nonpolar to polar media (see Table 1), it should be possible to record emission spectra to harness this reporter function so as to monitor polarity changes in the immediate local environment of single molecules. ${ }^{33}$ 
TABLE 4: Sensitivity of DCDHF-N-6 Fluorescence Lifetime $\left(\tau_{\mathrm{F}}\right)$ to Different Environments ${ }^{a}$

\begin{tabular}{ccc}
\hline solvent & $\Phi_{\mathrm{F}}$ & $\tau_{\mathrm{F}}[\mathrm{TPE}](\mathrm{ns})$ \\
\hline ethanol & 0.017 & $<0.22$ \\
toluene & 0.85 & $2.7[2.5]$ \\
PMMA & 0.98 & $3.2[2.9]$
\end{tabular}

${ }^{a}$ Values in brackets are fluorescence lifetimes with two-photon excitation. The lifetime in ethanol is shorter than the instrument response. Measured lifetime values are $\pm 0.1 \mathrm{~ns}$. (Quantum yields from Table 2 are included for comparing trends.)

Fluorescence-lifetime imaging microscopy ${ }^{34}$ is another imaging modality available using DCDHF molecules. Table 4 shows that both the one-photon excited and two-photon excited (in brackets) fluorescence lifetimes of bulk DCDHF-N-6 samples depend on the solvent or environment in which the fluorophore resides. The one-photon lifetime increases from below the instrument response time in polar ethanol to a few nanoseconds in nonpolar toluene and even higher in PMMA; the two-photon lifetime exhibits a similar increase going from solution to polymer. The increase in lifetime can be correlated to the increase in quantum yield and is most likely the result of suppressing the nonemissive TICT relaxation channel in toluene and PMMA. This property could be used to follow dynamic changes in local environment on the single-molecule level or to map different regions within a sample, using fluorescence lifetime as contrast.

3.2. Single-Molecule Properties. A strong test of the utility of a fluorophore is its ability to be imaged at the single-molecule level, which requires bright fluorescence, weak coupling with dark states, and robust photostability. The acene DCDHFs are superb single-molecule emitters: for example, single copies of DCDHF-A-6 and DCDHF-N-6 in films of aqueous protein gels are easily visible as shown in Figure 4, parts A and B, widefield epifluorescence images of typical samples.

To better characterize the photostability of a single-molecule emitter using one simple parameter, we recorded the distribution of the number of photons emitted from single fluorophores before photobleaching, $N_{\text {tot,emitted }}$ (see Table 1). This parameter assesses a fundamental property of the emitter because it should not depend on excitation intensity (in the absence of saturation or nonlinear effects): if the pumping intensity is selected to produce a desired emission rate, this parameter assesses how long a molecule will survive on average. From wide-field epifluorescence movies, 193 DCDHF-N-6 and 135 DCDHFA-6 single-molecule traces (similar to Figure 4C, inset) were recorded, inspected for digital bleaching, background-subtracted, and integrated to build a distribution (Figure 4C). These $N_{\text {tot,detected }}$ distributions exhibit the expected exponential shape for a first-order photobleaching process, and single-exponential fits yield 130000 photons detected per DCDHF-N-6 molecule on average and 226000 photons detected per DCDHF-A-6. Converting to the number emitted using the detection efficiency $D$, we find $N_{\text {tot,emitted }}=1.4 \times 10^{6}$ photons emitted per DCDHFN-6 molecule on average and $2.2 \times 10^{6}$ per DCDHF-A-6, which approach the values of DCDHF-P-6 and rhodamine 6G $\left(2.4 \times 10^{6}\right.$ and $1.9 \times 10^{6}$ photons emitted per molecule, respectively), ${ }^{15,24}$ both of which are demonstrably good singlemolecule fluorophores.

3.3. Bulk Photostability. The single-molecule $N_{\text {tot,emitted }}$ value for DCDHF-N-6 is comparable to the value determined using the bulk photobleaching behavior of high-concentration samples of DCDHF-N-6 in PMMA. To perform the ensemble-averaged measurement, plots of integrated, background-subtracted emission intensities versus time for bulk samples were fit using two
A

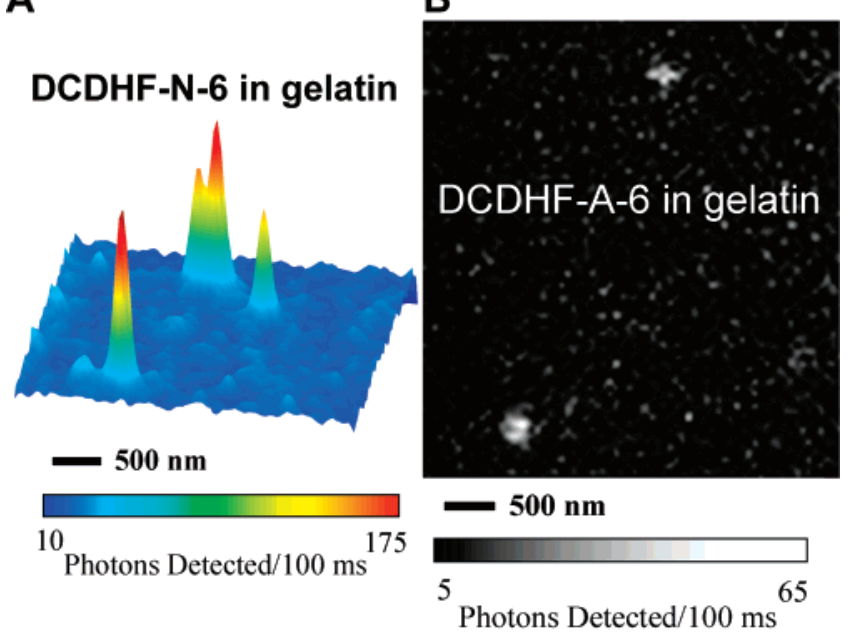

C

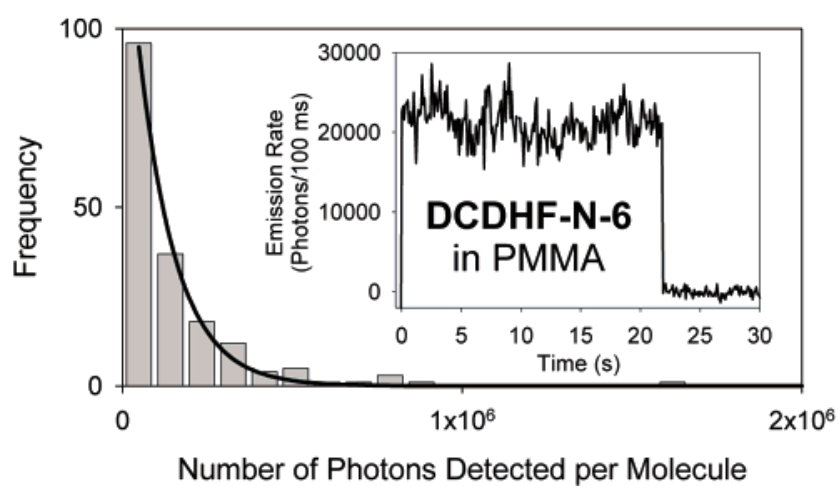

Figure 4. (A) Surface plot of emission from single DCDHF-N-6 molecules in a gelatin film, with $2 \times 2$ Gaussian smoothing. For this epifluorescence image, the excitation wavelength was $532 \mathrm{~nm}$, the intensity at the sample was approximately $0.25 \mathrm{~kW} / \mathrm{cm}^{2}$, and the integration time was $100 \mathrm{~ms}$ per frame. Pixel intensities in images were corrected for the dark offset count rate of the camera, then converted to photons detected. (B) Single DCDHF-A-6 molecules imaged in a gelatin film; the excitation wavelength was $594 \mathrm{~nm}$, the intensity at the sample was approximately $0.45 \mathrm{~kW} / \mathrm{cm}^{2}$, and the integration time was $100 \mathrm{~ms}$. (C) Histogram of total photons detected from 193 different DCDHF-N-6 molecules in a PMMA film. $N_{\text {tot,detected }}$ is the exponential parameter of a single-exponential fit (solid line). Inset: The spatially integrated fluorescence intensity time trace of a representative individual molecule. The reported intensity is background-subtracted and converted to photons emitted. Emission terminates at $22 \mathrm{~s}$ due to photobleaching. Very few molecules exhibited any blinking on the $100 \mathrm{~ms}$ integration time scale of the measurement, which is consistent with what has been reported for other members of the class of DCDHF fluorophores (ref 15).

exponentials, and the time constant $\tau_{\mathrm{B}}$ was extracted from these photobleaching curves. ${ }^{35}$ The number of photons emitted was calculated from the inverse of the photobleaching quantum yield using the absorption cross section $\sigma_{532}$ of DCDHF-N-6 $(1.8 \times$ $\left.10^{-16} \mathrm{~cm}^{2}\right)$, the illumination intensity $I_{532}$, and the quantum yield:

$$
\begin{aligned}
N_{\text {tot }, \text { emitted }}=N_{\mathrm{abs}} \Phi_{\mathrm{F}}= & \\
& R_{\mathrm{abs}} \tau_{\mathrm{B}} \Phi_{\mathrm{F}}=\sigma_{532} I_{532}\left(\frac{532 \mathrm{~nm}}{h c}\right) \tau_{\mathrm{B}} \Phi_{\mathrm{F}}
\end{aligned}
$$

where $N_{\mathrm{abs}}$ is the number of photons absorbed, $R_{\mathrm{abs}}$ is the rate of photon absorption, $h$ is Planck's constant, and $c$ is the speed of light in vacuum. This technique is independent of the photon losses in the microscope, so it is a separate corroboration of the single-molecule measurement. A bulk measurement yielded 
A

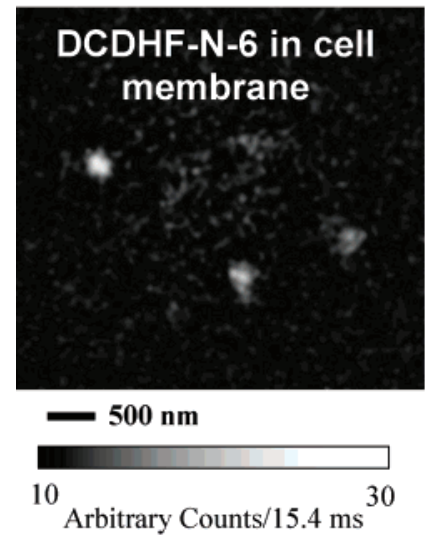

B

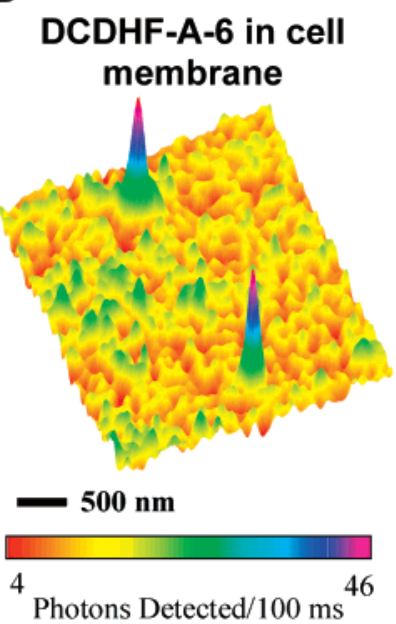

Figure 5. Epifluorescence images of acene DCDHFs in living $\mathrm{CHO}$ cells. (A) Image of single DCDHF-N-6 molecules diffusing in a region of a CHO plasma membrane, with $2 \times 2$ Gaussian smoothing. The excitation wavelength was $532 \mathrm{~nm}$, the intensity at the sample was approximately $2 \mathrm{~kW} / \mathrm{cm}^{2}$, and the integration time was $15.4 \mathrm{~ms}$ per frame. Detailed information and analysis of DCDHF-N-6 in cell membranes can be found in ref 22. (B) Surface plot of emission from single copies of DCDHF-A-6 in a CHO cell membrane, with $3 \times 3$ Gaussian smoothing. The excitation wavelength was $594 \mathrm{~nm}$, the intensity at the sample was approximately $0.75 \mathrm{~kW} / \mathrm{cm}^{2}$, and the integration time was $100 \mathrm{~ms}$.

$N_{\text {tot,emitted }}=2.6 \times 10^{6}$ photons emitted per DCDHF-N-6 molecule, comparable to the single-molecule value of $1.4 \times$ $10^{6}$; if anything, the single-molecule value may be an underestimate. As an independent check of this method of calculating

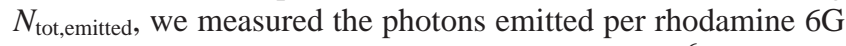
molecule in polyvinyl alcohol to be $1.4 \times 10^{6}$, which is comparable to the published ${ }^{24}$ value of $1.9 \times 10^{6}$ photons emitted per rhodamine $6 \mathrm{G}$ molecule in ethanol.

To compare the photostability of the acene DCDHFs to other commonly used cellular fluorophores in a cell-like model environment, we measured the photobleaching quantum yield $\left(\Phi_{\mathrm{B}}\right)$ in an aqueous protein gel. $\Phi_{\mathrm{B}}$ is the probability that a single molecule will photobleach after absorbing a photon, so the smaller the value of $\Phi_{\mathrm{B}}$, the more total photons are available for detection. We define the photobleaching quantum yield as

$$
\Phi_{\mathrm{B}}=\frac{n_{\text {bleach }}}{N_{\mathrm{abs}}}=\frac{R_{\text {bleach }}}{R_{\mathrm{abs}}}=\frac{1}{\tau_{\mathrm{B}} R_{\mathrm{abs}}}=\frac{1}{\tau_{\mathrm{B}} \sigma_{\lambda} I_{\lambda}\left(\frac{\lambda}{h c}\right)}
$$

where $n_{\text {bleach }}$ is the number of molecules bleached after a measured time and $R_{\text {bleach }}$ is the bleaching rate. The time constant $\tau_{\mathrm{B}}$ was found by fitting the bleaching curves of the different fluorophores using three exponentials with a vertical offset to account for the background; $\tau_{\mathrm{B}}$ is the weighted average of the three time constants. Each fluorophore was measured at several different illumination intensities, all below saturation. Our measured values for R6G and Texas Red $\Phi_{\mathrm{B}}$ are similar to their respective literature values in water. ${ }^{24}$ Average values for all measured $\Phi_{\mathrm{B}}$ are displayed in Table 5; the acene DCDHFs prove to be many times more photostable than rhodamine $6 \mathrm{G}$, Texas Red, and fluorescein in this aqueous environment.

The acene DCDHFs not only are resilient to photobleaching, they also resist entering long-lived dark states: most DCDHFN-6 and DCDHF-A-6 molecules exhibited little or no blinking on the time scale of our experiments (i.e., $100 \mathrm{~ms}$ integration
TABLE 5: Acene DCDHFs Perform Well in a Comparison of Photobleaching Quantum Yields in Aqueous Gelatin

\begin{tabular}{lc}
\hline fluorophore & $\Phi_{\mathrm{B}}\left(10^{-6}\right)$ \\
\hline DCDHF-N-6 & 1.92 \\
DCDHF-A-6 & 10.5 \\
R6G & 12.3 \\
Texas Red & 36.7 \\
fluorescein & 56.6
\end{tabular}

time, movies lasting tens of seconds), as seen in the sample time-trace inset of Figure 4C.

3.4. Cellular Imaging. The ultimate test of our fluorophores is whether they can be imaged on the single-molecule level in a cellular environment long enough to report on some process of interest. Recently, we successfully used several different DCDHF derivatives as fluorescent lipid analogs as tracers to probe cellular membrane dynamics. ${ }^{22}$ Single copies of the naphthyl derivative (both DCDHF-N-6 and DCDHF-N-12, a version with longer alkyl chains on the amine donor) were visualized diffusing in the plasma membrane of mammalian $\mathrm{CHO}$ cells (Figure 5A). We were also able to image single copies of DCDHF-A-6 in CHO cell membranes (Figure 5B). In the future, with further optimization of the DCDHF class, we hope to design additional high-performance reporter molecules capable of detection at the single-molecule level in cells, but already DCDHF-N-6 emits as many photons and can be imaged with a signal-to-noise ratio comparable to that of a popular lipid-analog fluorophore, Tritc-DHPE (a tetramethylrhodamine derivative), as reported in detail in ref 22 .

\section{Conclusion}

This work has described two acene relatives of the earlierreported DCDHF-P-6 molecule, DCDHF-N-6 and DCDHFA-6, which exhibit strong fluorescence upon pumping at the longer wavelengths that are necessary for single-molecule imaging in cells. These two long-wavelength fluorophores also exhibit the strong emission and weak dark states which give rise to single-molecule properties approaching those of DCDHFP-6 and rhodamine 6G when imbedded in a PMMA matrix and superior photostability in an aqueous protein environment.

Further experiments involving DCDHF-N-6 and DCDHFA-6 can take advantage of other properties of the DCDHF class of fluorophores that these acene derivatives possess, such as measurable shifts in emission wavelength or fluorescence lifetime with variations in local polarity. Such experiments include protein labeling using maleimide covalent binding and FRET pairing with other fluorophores or other DCDHF derivatives. In some biological experiments, it may be necessary to impart better water solubility to these fluorophores, which can be achieved by adding alcohol, carboxylic acid, or sulfonic acid functional groups to the structure (either off the donor or acceptor). ${ }^{36}$ These first results - minimized background fluorescence from free fluorophores in solution, long-wavelength absorption and emission, high photostability in aqueous environments, and large solvatochromism-demonstrate that acenelinked DCDHF fluorophores are high-quality single-molecule emitters for cellular imaging.

Acknowledgment. The authors thank Yuan Li for assistance with calculations of the electronic structure of DCDHF-N-6, Dr. Frank Jäckel for ellipsometry measurements, Kit Werley for his suggestion to use eq 2, and Professor Jörg Enderlein for discussion about objective collection efficiency. This research was supported in part by the National Institutes of Health through the NIH Roadmap for Medical Research, Grant No. 1P20-HG003638. 


\section{References and Notes}

(1) Sako, Y.; Yanagida, T. Review: Single-Molecule Visualization in Cell Biology. Nat. Rev. Mol. Cell Biol. 2003, 4, SS1-SS5.

(2) Moerner, W. E. Optical Measurements of Single Molecules in Cells. TrAC, Trends Anal. Chem. 2003, 22, 544-548.

(3) Tinnefeld, P.; Sauer, M. Branching Out of Single-Molecule Fluorescence Spectroscopy: Challenges for Chemistry and Influence on Biology. Angew. Chem., Int. Ed. 2005, 44, 2642-2671.

(4) Schutz, G. J.; Kada, G.; Pastushenko, V. P.; Schindler, H. Properties of Lipid Microdomains in a Muscle Cell Membrane Visualized by Single Molecule Microscopy. EMBO J. 2000, 19, 892-901.

(5) Vrljic, M.; Nishimura, S. Y.; Brasselet, S.; Moerner, W. E.; McConnell, H. M. Translational Diffusion of Individual Class II MHC Membrane Proteins in Cells. Biophys. J. 2002, 83, 2681-2692.

(6) Lakadamyali, M.; Rust, M. J.; Babcock, H. P.; Zhuang, X. Visualizing Infection of Individual Influenza Viruses. Proc. Natl. Acad. Sci. U.S.A. 2003, 100, 9280-9285.

(7) Kim, S. Y.; Gitai, Z.; Kinkhabwala, A.; Shapiro, L.; Moerner, W. E. Single Molecules of the Bacterial Actin MreB Undergo Directed Treadmilling Motion in Caulobacter Crescentus. Proc. Natl. Acad. Sci. U.S.A. 2006, 103, 10929-10934.

(8) Xie, X. S.; Yu, J.; Yang, W. Y. Living Cells as Test Tubes. Science 2006, 312, 228-230.

(9) Jung, C.; Muller, B. K.; Lamb, D. C.; Nolde, F.; Mullen, K.; Brauchle, C. A New Photostable Terrylene Diimide Dye for Applications in Single Molecule Studies and Membrane Labeling. J. Am. Chem. Soc. 2006, 128, 5283-5291.

(10) Toutchkine, A.; Kraynov, V.; Hahn, K. Solvent-Sensitive Dyes to Report Protein Conformational Changes in Living Cells. J. Am. Chem. Soc. 2003, $125,4132-4145$

(11) Jaiswal, J. K.; Simon, S. M. Potentials and Pitfalls of Fluorescent Quantum Dots for Biological Imaging. Trends Cell Biol. 2004, 14, 497.

(12) Chudakov, D. M.; Lukyanov, S.; Lukyanov, K. A. Fluorescent Proteins as a Toolkit for in Vivo Imaging. Trends Biotechnol. 2005, 23 , 605-613.

(13) Lee, T.; Gonzales, J. I.; Zheng, J.; Dickson, R. Single-Molecule Optoelectronics. Acc. Chem. Res. 2005, 38, 534-541.

(14) Willets, K. A.; Nishimura, S. Y.; Schuck, P. J.; Twieg, R. J.; Moerner, W. E. Nonlinear Optical Chromophores as Nanoscale Emitters for Single-Molecule Spectroscopy. Acc. Chem. Res. 2005, 38, 549-556.

(15) Willets, K. A.; Ostroverkhova, O.; He, M.; Twieg, R. J.; Moerner, W. E. New Fluorophores for Single-Molecule Spectroscopy. J. Am. Chem. Soc. 2003, 125, 1174-1175.

(16) Willets, K. A.; Callis, P. R.; Moerner, W. E. Experimental and Theoretical Investigations of Environmentally Sensitive Single-Molecule Fluorophores. J. Phys. Chem. B 2004, 108, 10465-10473.

(17) Schuck, P. J.; Willets, K. A.; Fromm, D. P.; Twieg, R. J.; Moerner, W. E. A Novel Fluorophore for Two-Photon-Excited Single-Molecule Fluorescence. Chem. Phys. 2005, 318, 7-11.

(18) Grabowski, Z. R.; Rotkiewicz, K.; Rettig, W. Structural Changes Accompanying Intramolecular Electron Transfer: Focus on Twisted Intramolecular Charge-Transfer States and Structures. Chem. Rev. 2003, 103, $3899-4031$

(19) Jacobson, A.; Petric, A.; Hogenkamp, D.; Sinur, A.; Barrio, J. R 1,1-Dicyano-2-[6-(dimethylamino)naphthalen-2-yl]propene (DDNP): A Solvent Polarity and Viscosity Sensitive Fluorophore for Fluorescence Microscopy. J. Am. Chem. Soc. 1996, 118, 5572-5579.
(20) Harms, G. S.; Cognet, L.; Lommerse, P. H. M.; Blab, G. A.; Schmidt, T. Autofluorescent Proteins in Single-Molecule Research: Applications to Live Cell Imaging Microscopy. Biophys. J. 2001, 80, 23962408.

(21) Lu, Z.; Lord, S. J.; Wang, H.; Moerner, W. E.; Twieg, R. J. LongWavelength Analogue of PRODAN: Synthesis and Properties of Anthradan, a Fluorophore with a 2,6-Donor-Acceptor Anthracene Structure. J. Org. Chem. 2006, 71, 9651-9657.

(22) Nishimura, S. Y.; Lord, S. J.; Klein, L. O.; Willets, K. A.; He, M.; Lu, Z. K.; Twieg, R. J.; Moerner, W. E. Diffusion of Lipid-Like SingleMolecule Fluorophores in the Cell Membrane. J. Phys. Chem. B 2006, 110, 8151-8157.

(23) Lakowicz, J. R. Principles of Fluorescence Spectroscopy; Springer: New York, 2006.

(24) Soper, S. A.; Nutter, H. L.; Keller, R. A.; Davis, L. M.; Shera, E. B. The Photophysical Constants of several Fluorescent Dyes Pertaining to Ultrasensitive Fluorescence Spectroscopy. Photochem. Photobiol. 1993, 57 , 972-977.

(25) Moerner, W. E.; Fromm, D. P. Methods of Single-Molecule Fluorescence Spectroscopy and Microscopy. Rev. Sci. Instrum. 2003, 74, $3597-3619$

(26) Zander, C., Enderlein, J., Keller, R. A., Eds. Single-Molecule Detection in Solution: Methods and Applications; Wiley-VCH: Berlin, 2002.

(27) Gubler, U.; He, M.; Wright, D.; Roh, Y.; Twieg, R. J.; Moerner, W. E. Monolithic Photorefractive Organic Glasses with Large Coupling Gain and Strong Beam Fanning. Adv. Mater. 2002, 14, 313-317.

(28) He, M.; Twieg, R.; Ostroverkhova, O.; Gubler, U.; Wright, D.; Moerner, W. E. Dicyanomethylenedihydrofuran Photorefractive Materials. Proc. SPIE-Int. Soc. Opt. Eng. 2002, 4802, 9-20.

(29) Wang, H.; Lu, Z.; Lord, S.; Willets, K.; Bertke, J.; Bunge, S.; Moerner, W.; Twieg, R. The Influence of Tetrahydroquinoline Rings in Dicyanomethylenedihydrofuran (DCDHF) Single-Molecule Fluorophores. Tetrahedron 2007, 63, 103-114.

(30) Picard, J. P.; Elyusufi, A. A.; Calas, R.; Dunogues, J.; Duffaut, N. Enamines of Acylsilanes: An Easy Access from Alpha-Siloxynitriles. Organometallics 1984, 3, 1660-1665.

(31) Weast, R. C., Ed. CRC Handbook of Chemistry and Physics; CRC Press: Boca Raton, FL, 1989.

(32) Gordon, A. J.; Ford, R. A. The Chemist's Companion; Wiley: New York, 1972.

(33) Hou, Y.; Bardo, A. M.; Martinez, C.; Higgins, D. A. Characterization of Molecular Scale Environments in Polymer Films by Single Molecule Spectroscopy. J. Phys. Chem. B 2000, 104, 212-219.

(34) Siegel, J.; Elson, D. S.; Webb, S. E. D.; Lee, K. C. B.; Vlanclas, A.; Gambaruto, G. L.; Leveque-Fort, S.; Lever, M. J.; Tadrous, P. J.; Stamp, G. W. H.; Wallace, A. L.; Sandison, A.; Watson, T. F.; Alvarez, F.; French, P. M. W. Studying Biological Tissue with Fluorescence Lifetime Imaging: Microscopy, Endoscopy, and Complex Decay Profiles. Appl. Opt. 2003 , 42, 2995-3004.

(35) Peterman, E. J. G.; Brasselet, S.; Moerner, W. E. The Fluorescence Dynamics of Single Molecules of Green Fluorescent Protein. J. Phys. Chem. A 1999, 103, 10553-10560.

(36) Wang, H.; Lu, Z.; Lord, S. J.; Moerner, W. E.; Twieg, R. J. Modifications of DCDHF Single Molecule Fluorophores to Impart Water Solubility. Tetrahedron Lett. 2007, 48, 3471-3474. 